\title{
Article \\ miCampoApp, a Participatory Research Tool for Agroecology
}

\author{
Stephane Dupas ${ }^{1,2} \mathbb{D}$, Cesar Alejandro Antolinez ${ }^{1 *}$ \\ 1 Université Paris-Saclay, CNRS, IRD, UMR Évolution, Génomes, Comportement et Écologie, 91198, \\ Gif-sur-Yvette, France \\ 2 Departmento de Ciencias Biologicas, Universidad de Los Andes, Bogota, Colombia \\ * Correspondence: Stephane.Dupas@ird.fr; Tel.: +57-301- 264-3792
}

\begin{abstract}
Agroecology, defined as the ecological science of food production is also as practical approach to design food production systems based on local concerted solutions that aim to promote synergy among the diversity of human and non human food systems elements. These two facets makes agroecology a good candidate for participatory research. Information technology should help using this information for the production of structured scientific knowledge. In this respect, there is a need for information technology that is adaptive to encompass the diversity of within and between systems and that provide benefit to farmers that feed it with data. We present MiCampoApp, a webapp that aims to join participatory research and certification in agroecology, with two roles, the farmer and the administrator. The idea to join participatory research for decision support with certification for market differentiation in single system is that much of the certification data harbors information for scientific research, and this incentivize data collection for the interest of the farmer and the community. The administrator create a model for data collection in order to solve a research question of interest for a communities or to produce traceability information to feed participatory guarantee system in a community. The farmer collects information using simple icons and produces traceability pages for research or certification purposes.
\end{abstract}

Keywords: agroecology; participatory research; web application; traceability

\section{Introduction}

Agroecology, is a discipline and a movement [1].As a discipline is can be defined as the ecological science of food production [2]. Agroecology is also a movement and a set of principles of action aiming to drive the transformation of food system toward sustainability [1]. Among its principles of action is the choice to build concerted solutions, promoting diversity and synergy among ecosystem components, based on local observations and knowledge [3]. Agroecological diversification and local solutions therefore harbors high value in terms of participatory research on agroecosystems components and their interactions. Yet putting together this knowledge and the diversity of local solutions, and testing agroecological hypotheses regarding synergies and diversity requires organizing a common agroecological dataset [4]. There is however a lack of traceability of agroecological practices [5](Warner et al 2008). This lack of traceability is not only a problem for science, it is a problem of market differentiation. Agroecology local solutions do not necessarily meet certification standards, and costs of standard certification are unaffordable in particular for low income farmers [6? ,7](FAO 2007, Altieri and Nicholls, 2008, IFAD-ORG 2004). Agroecology to the difference of organic agriculture is not a production system but a set of principles to build cooperative diversified and resilient food systems. It is therefore difficult to standardize data to be collected to certify agroecological farms and products. In order to make certification and traceability more affordable to small holder, and to assess agroecology principles, participatory guarantee systems have been promoted [8] and developed from users, based on sharing ethical values and certifying by the active participation among farmers or between farmers and consumers rather than indirectly and independently form 
third party company [9]. This partially reduces the cost of agro-ecology certification but still demands organizational costs. These two issues of participatory research and market differentiation agroecology can share the common objective of identifying indicator variables linked to sustainability of food systems. The possibility to collect and share information by the internet can be seen as an opportunity to address traceability problems [10]. Yet there is a need for information systems that allow a diversity of production and transformation, distribution and consumption models to be implemented and traced for market and research purposes. There is a need of a data platform able to adapt to each agroecological system and practices to combine agroecological principles and certification needs. We propose in this article a web application, a generic tool that permits the user to develop its own data collection system with uploaded icons to simplify interface to be able to feed with participatory data that can be tagged and published in traceability web pages links for research evaluation and market differentiation. The app includes modules for sensors and prediction of risks.

\section{Web app functionalities}

The web app exchanges information between tree user types, administrator that create models, the farmers and the public (Figure 1).

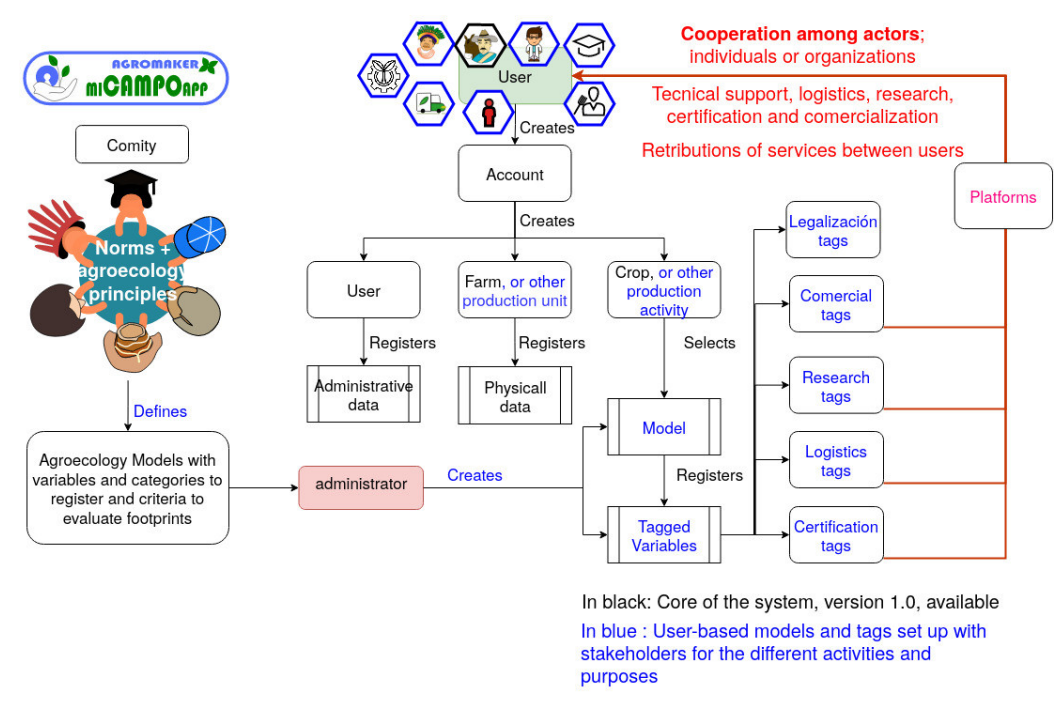

Figure 1. Workflow of miCampo App.

\subsection{Administrator functionalities}

The administrator (Figure 2) can log in to the web page and,

1. Edit his profile

2. Create data models ("Cultivos"), categories within data models ("categorias"), and create variables within categories. Available variable types are : Boolean, numeric, text, percent, range, categories selection, categories based on image selection, categories associated with quantities. Data models, categories, and variables are attributed simple icons uploaded to the system. The system count with a large number of icons that allow to represent agroecological information in its different dimensions of practices, crops (89 crops are graphically represented), farm characterization for certification, and crop pest and disease observations for research. Each variable is associated with help pages (links, images, text alimented by administrator) to accompany users data collection.

3. Activate or deactivates variables and categories so that users can or cannot collect data for these variables. 
4. Generate TAGs ("Etiquetas") and add these tags in the variable edition panel. TAGs are selection of variables that can be published as web pages by users for traceability purposes (research, or market differentiation).

5. Create models of sensors with the format of data transmission that can be used by users to connect IOTs to the system .

6. Activate or desactivate sensors registered by users.

7. Generate posts in the BLOG web pages for the system that can be used as help pages for users of the system. And activate or desactivate web pages of any user.

8. Provide registered user administrator role

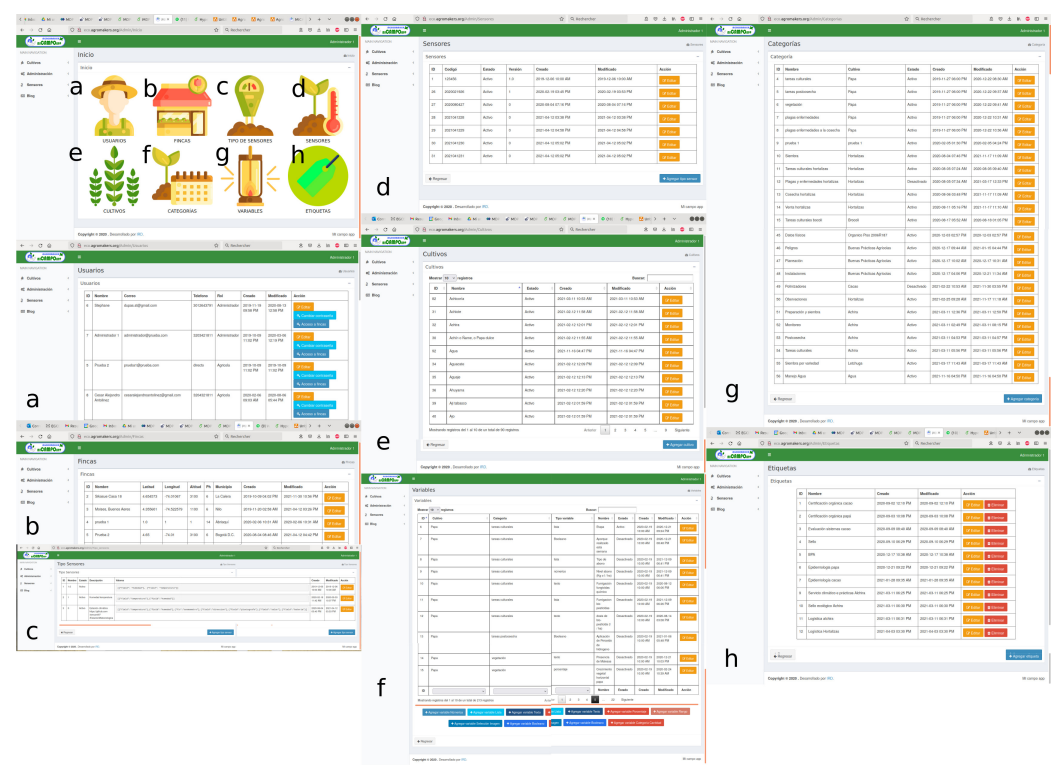

Figure 2. System administration. The letters represent hyperlinks

\subsection{Farmer functionalities}

The farmer can

1. Register as user

2. Register and edit its production unit ("Finca") (Figure 3)

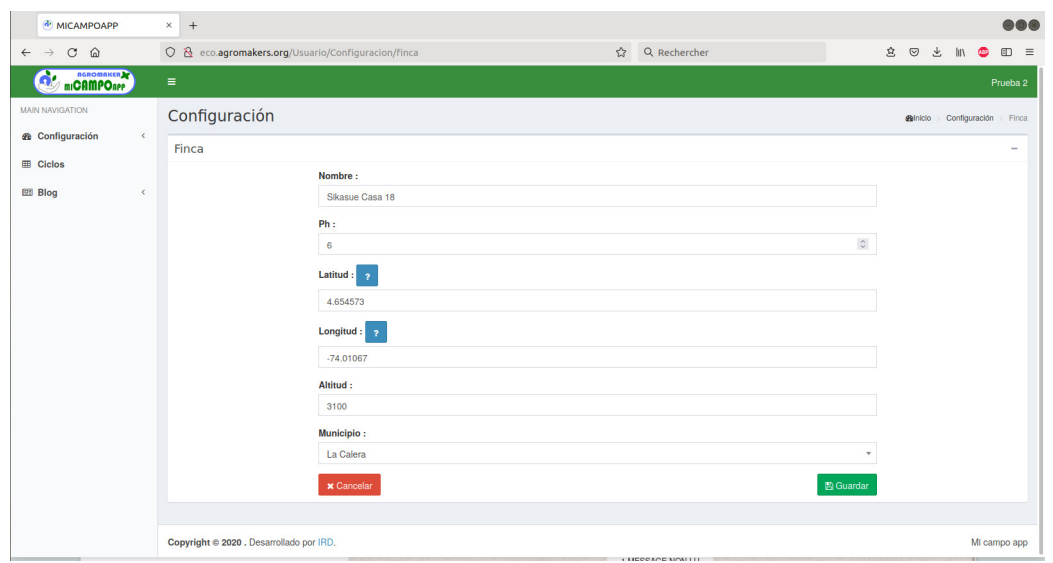

Figure 3. Production unit (Finca) registering.

3. Register and edit its functional units ("Parcela") (Figure 4) 


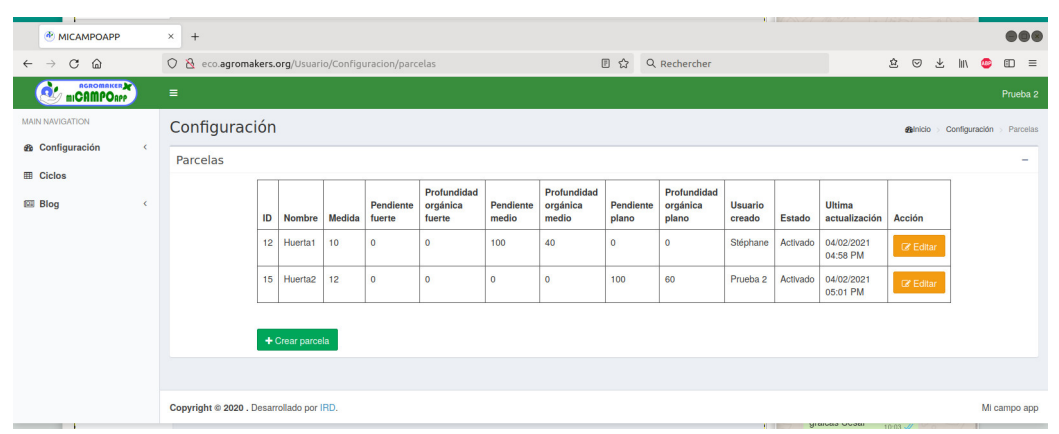

Figure 4. Functional unit (Parcela) registering.

4. Visualize, edit and create (Figure 5) production cycles as defined as data models by administrator.

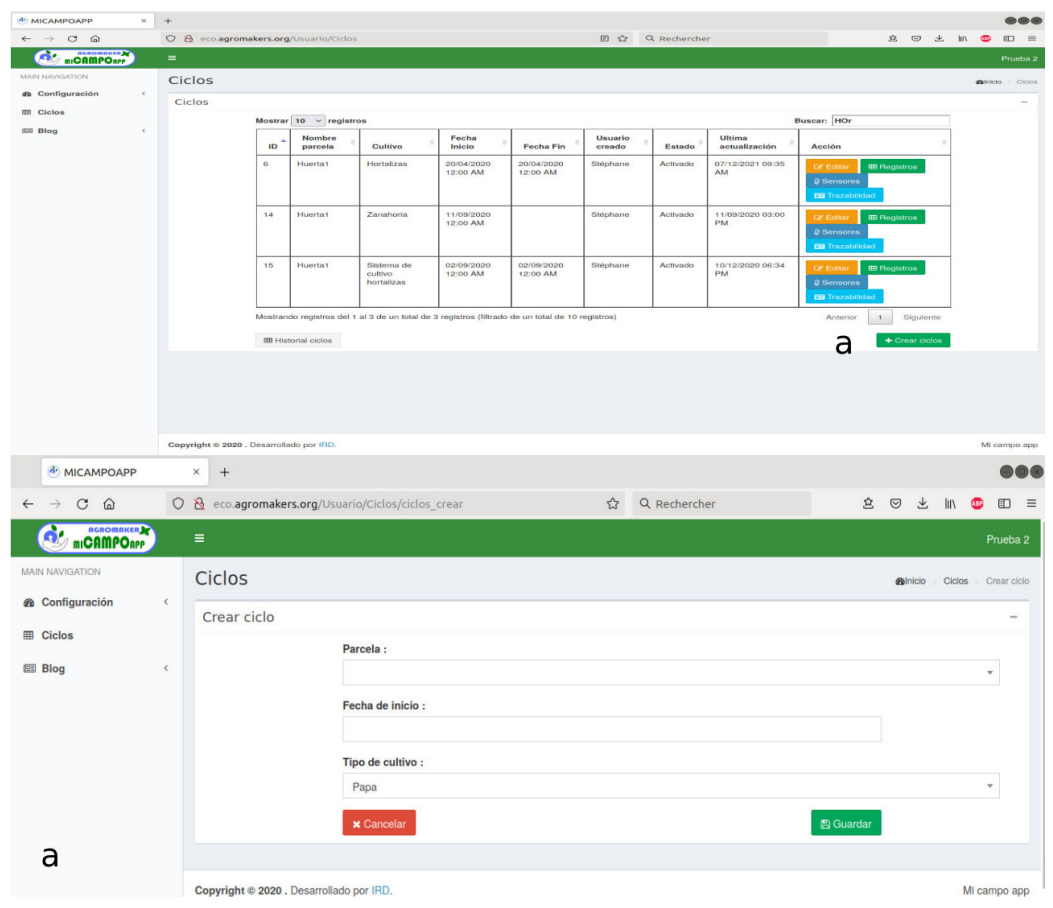

Figure 5. Cycle selection for editing (Editar), registering (Registros), sensor edition (Sensores), labelling (Trazabilidad), or creating (crear cycle).The letters represent hyperlinks.

The following models are already available:

(a) Crop production cycles. They include data on management practices associated to these crops (planting, hilling, pruning, spraying, harvesting...), and observations including lists of principal pests and diseases observed on these crops.

i. The vegetables associations (hortalizas) cycles (Figure 6) 


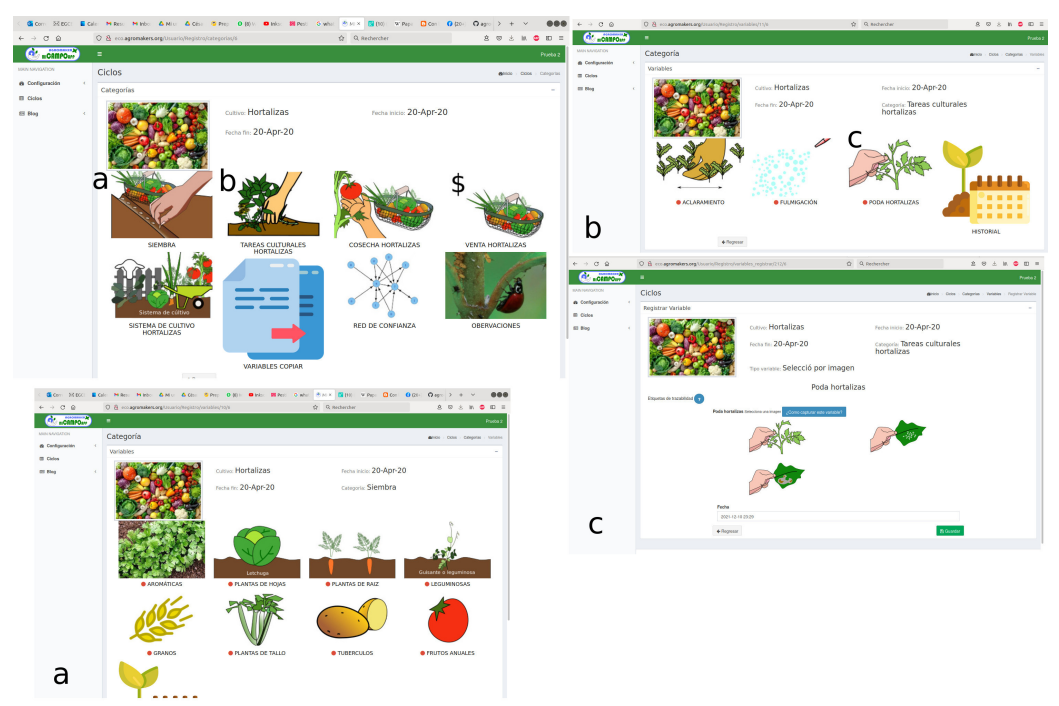

Figure 6. Vegetables cycle categories with variables within the two first categories, and registering of plant pruning variable.The letters represent hyperlinks.

ii. The cocoa (cacao) cycles (Figure 7). The cacao model aims to collect information of cacao crops and contains tags for certification and evaluation of practices. Cacao certification costs is a major limitation for improving practices and have access to appropriate market prices in south american countries (IFAD-ORG 2004).

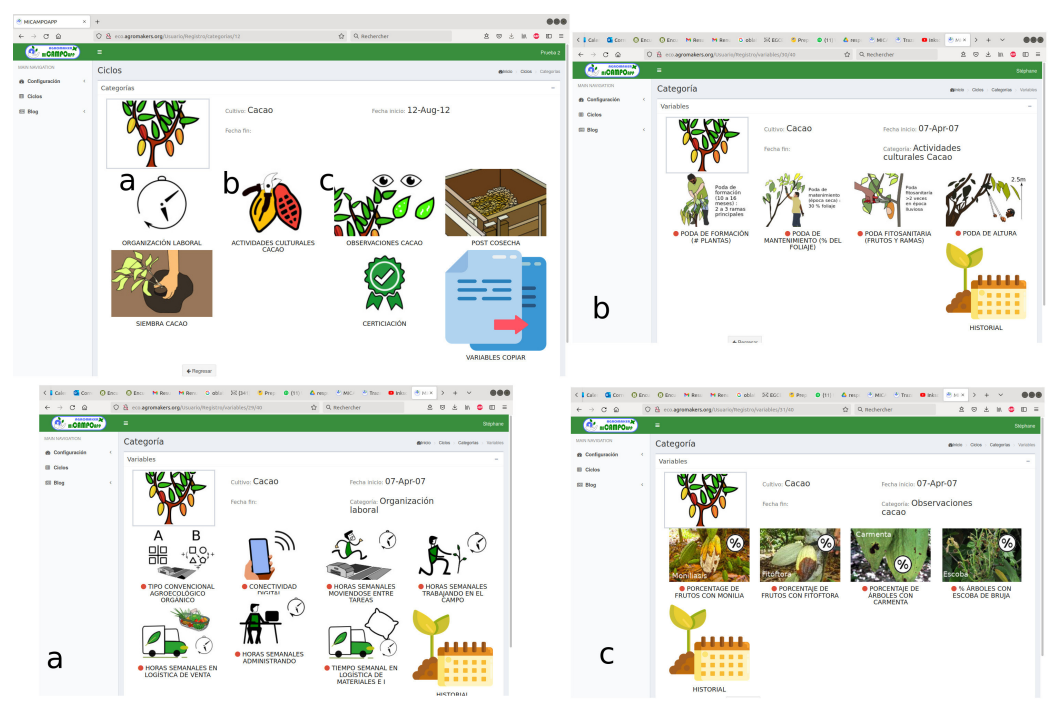

Figure 7. Cacao cycle categories with variables within the three first categories. The letters represent hyperlinks.

iii. The potato (papa) cycles (Figure 4c). The potato model aims to collect information of potato crops and epidemiological modelling. Potato crops are under strong epidemiological pressures that leads to overuse of pesticides. The models to predict risks of disease have been developed on temperate countries. We published in the web site prediction on potato blight units to aid farmer reducing pesticide use (agromakers.org:3838/interactiveBlightMap/). The potato cycle is aimed at improving this model through participatory science. 


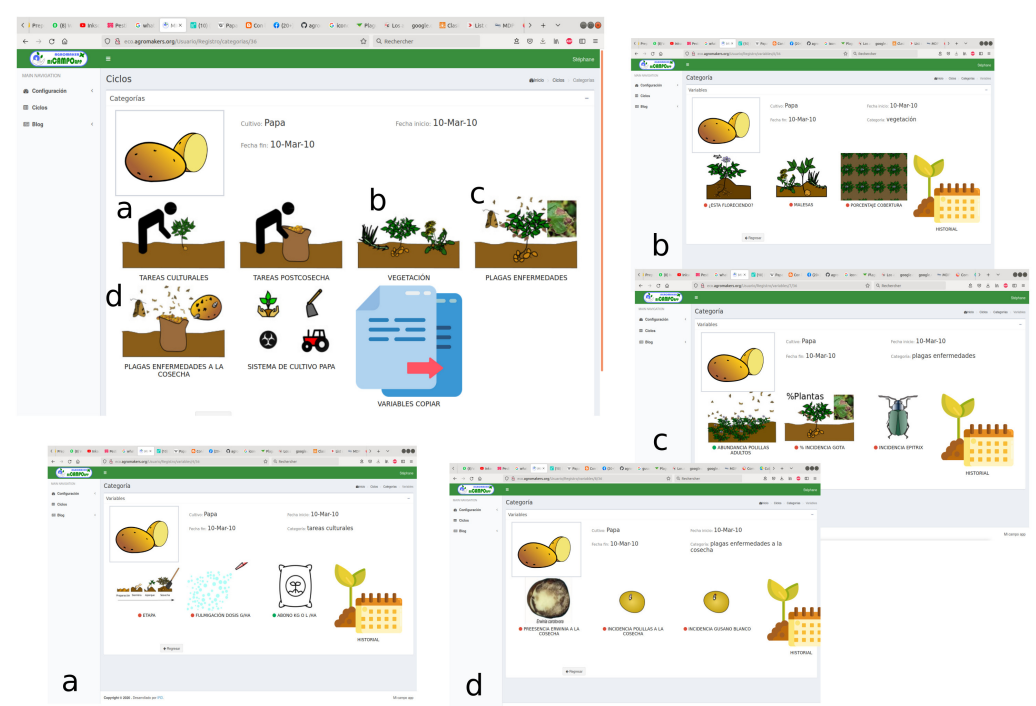

Figure 8. Potato cycle categories with categories and variables within the categories a, b, c and d.The letters represent hyperlinks.

iv. The canna (achira) cycles (Figure 9), allowing to include transformation tasks.

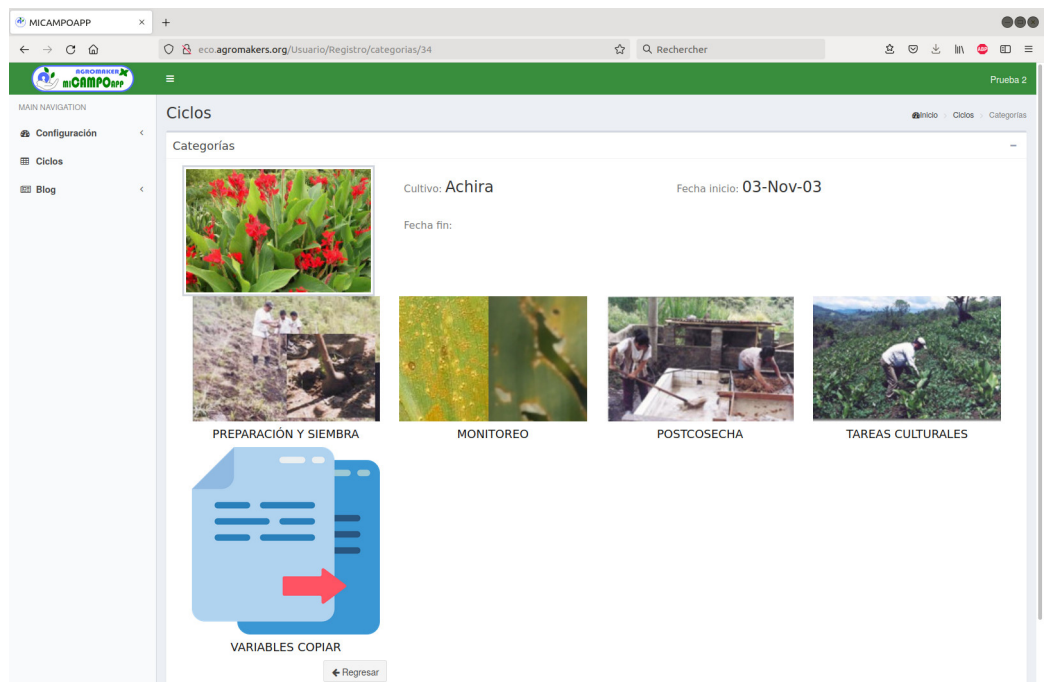

Figure 9. Canna cycle categories with categories.The letters represent hyperlinks.

v. Other possible cycles. We created cycles for 84 cultures with their icons. Categories an variables can be added to create traceability tools.

(b) Certification is a bottlneck of agroecology scaling (Altieri and Nicholls, 2008). Our tool aims at alowing to build data systems for certification on demand. We implemented a good farming practices (Buenas Prácticas agrícolas) cycle. It include 3 categories : category risks evaluation (peligros) includes icons for climatic risks, chemical risks, physic risks and biological risks evaluation ; category planification (planeación) includes icons for registering certification for water access, existence of risk map, water plan, aptitudes analysis, historical record of soil use; category infrastructures (instalaciones) includes caracterization of areas for workers, inputs storage, input preparation, product gathering, waste management. 


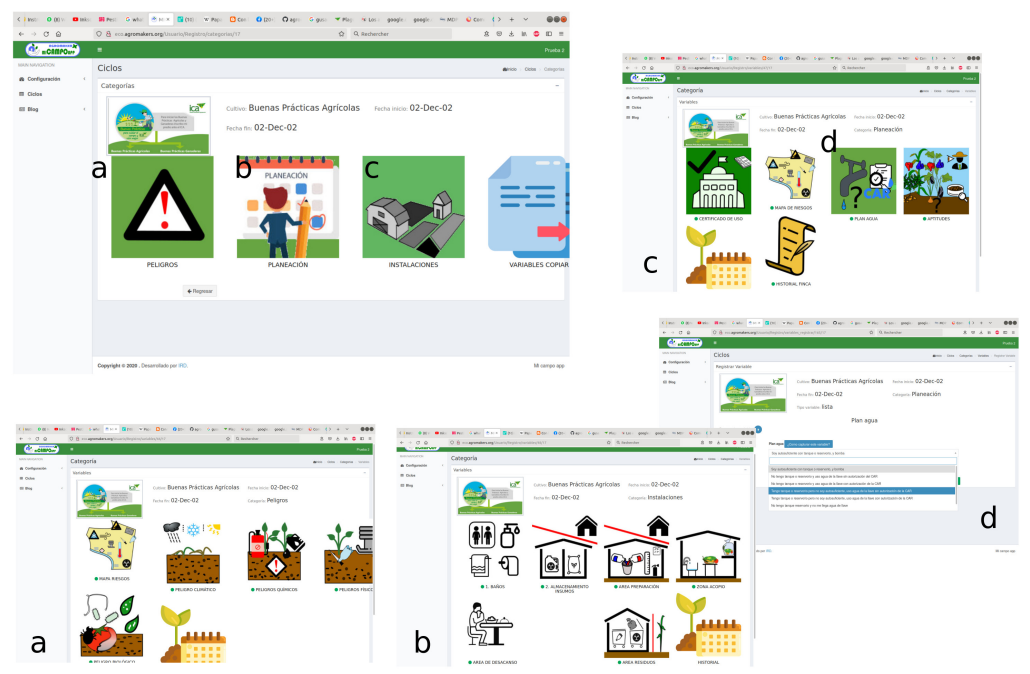

Figure 10. Certification cycle categories and variables

5. Add sensors that can be connected to the data base.

6. Generate traceability webpages on from cycles using variables selected in the tags. These can have certification, research or logistic purpose. The variables include in the tag

7. Generate BLOG web pages to produce more personal information on their production unit, their practices or other relevant information for the community.

\subsection{Code source}

The web app is developed in codeIgniter 2, available at github . upon request The current version is in spanish.

\subsection{Research protocol for participatory epidemiological modeling of potato blight}

We present a research protocol to use the web app for participatory research on potato blight epidemiological modeling for decision support on reduction of pesticide use. The irrational use of pesticide to control potato blight leads to major public heath problems in the Andean region [11]. Modeling can be used in temperate countries to develop early alert systems and reduce pesticide use ([12]). Other simple decision support can be used to accompany farmers decisions based on simple observations ([13]). However, such models may be misleading depending on microclimatic conditions, and when transferred to tropical regions due to differences in practices and climatic seasonality not accounted in the models ([14]). There is therefore a need for adaptation of models. The potato cycle and its traceablity pages (Figure 8) can be used in this purpose. This section may be divided by subheadings. It should provide a concise and precise description of the experimental results, their interpretation as well as the experimental conclusions that can be drawn.

\section{Discussion}

The web app is considered as an advance in agroeoclogical science and traceability in agroeoclogy since the administrator can adapt data collection system to the diversity of local solutions developed in agroecology, and allow cooperation between farmers and researchers in order to produce scientific knowledge. There does not seem to exist other such tool at the moment the that accomplish this function. This tool would allow to valorize the numerous research experiments performed by agroeocogical farmers. Nevertheless the application is a web app, that requires internet connection. This limits its use in rural due to gap in internet access ([15]). Its use as a traceability tool for market differentiation purpose is still in a research state since the traceability pages lack design work. Small agroecology farming, can have major contribution on the achievement of sustainable development goals (SDG), ([16]). The principle of agroecology that most contribution has on SDG, that emerges 
from the principle of building from local, is the diversification of its products an practices (Millenum Institute 2018). This diversification stabilizes small producers income related to variations in production and market prices ([17]). Our application aims to account for this diversity linking valorization in research and could be used for market differentiation in an adaptive way.

\section{Acknowledgments}

Author Contributions: Stéphane Dupas conceptualized the application and wrote the article, Alejandro Antolinez coded the source and mounted the webapp on the server

Funding: This work has been supported by IRD EGCE, International mixed laboratoy $\mathrm{BIO}_{I} \mathrm{NCA}$, andCGI ARPr Strengtheningtheevidencebase foraclimate - resilientandlow - carbonsmallholderagriculturethroughagroecolog

Institutional Review Board Statement:

Informed Consent Statement:

Data Availability Statement: Code source is available upon request at upon request

Acknowledgments: In this section you can acknowledge any support given which is not covered by the author contribution or funding sections. This may include administrative and technical support, or donations in kind (e.g., materials used for experiments).

Conflicts of Interest: Declare conflicts of interest or state "The authors declare no conflict of interest." Authors must identify and declare any personal circumstances or interest that may be perceived as inappropriately influencing the representation or interpretation of reported research results. Any role of the funders in the design of the study; in the collection, analyses or interpretation of data; in the writing of the manuscript, or in the decision to publish the results must be declared in this section. If there is no role, please state "The funders had no role in the design of the study; in the collection, analyses, or interpretation of data; in the writing of the manuscript, or in the decision to publish the results".

\section{References}

1. Wezel, A.; Bellon, S.; Doré, T.; Francis, C.; Vallod, D.; David, C. Agroecology as a science, a movement and a practice. Sustainable Agriculture 2009, 2, 27-43. doi:10.1007/978-94-007-03940_3.

2. Francis, C.; Lieblein, G.; Gliessman, S.; Breland, T.A.; Creamer, N.; Harwood, R.; Salomonsson, L.; Helenius, J.; Rickerl, D.; Salvador, R.; Wiedenhoeft, M.; Simmons, S.; Allen, P.; Altieri, M.; Flora, C.; Poincelot, R. Agroecology: The Ecology of Food Systems. Journal of Sustainable Agriculture 2003, 22, 99-118. doi:10.1300/J064v22n03.

3. FAO. Scaling Up Agroecology Initiative Transforming. Technical Report 12, 2018.

4. FAO. TAPE Tool for Agroecology Performance Evaluation 2019 - Process of development and guidelines for application. Test version; 2019.

5. Warner, K.D. Agroecology as participatory science: Emerging alternatives to technology transfer extension practice. Science Technology and Human Values 2008, 33, 754-777. doi: 10.1177/0162243907309851.

6. FAO. Agricultural management, marketing and finance working document 22. Technical report, 2007.

7. Altieri, M.A.; Nicholls, C.I. Scaling up agroecological approaches for food sovereignty in latin America. Development 2008, 51, 472-480. doi:10.1057/dev.2008.68.

8. FAO. Participatory Guarantee Systems (PGS) for sustainable local food systems. FAO Regional Offier for Asia and The Pacific 2018.

9. Ponce, N.L.C.; López, D.C.L. Aporte potencial de los sistemas participativos de garantía (SPG) a la soberanía alimentaria: caso SPG Caldas 2016-2017; Pontificia Universidad Javeriana, 2018.

10. Karippacheril, T.G.; Rios, L.D.; Srivastava, L. Global Markets, Global Challenges: Improving Food Safety and Traceability While Empowering Smallholders Through ICT. In ICT in Agriculture (Updated Edition): Connecting Smallholders to Knowledge, Networks, and Institutions; Number June, 2017; pp. 285-309. doi:10.1596/978-1-4648-1002-2.

11. Pradel, W.; Forbes, G.A.; Ortiz, O.; Cole, D.; Wanigartne, S. Use of the environmental impact quotient to estimate impacts of pesticide usage in three Peruvian potato production areas. 
12. Small, I.M.; Joseph, L.; Fry, W.E. Development and implementation of the BlightPro decision support system for potato and tomato late blight management. Computers and Electronics in Agriculture 2015, 115, 57-65. doi:10.1016/j.compag.2015.05.010.

13. Pérez, W.; Arias, R.; Taipe, A.; Ortiz, O.; Forbes, G.A.; Andrade-Piedra, J.; Kromann, P. A simple, hand-held decision support designed tool to help resource-poor farmers improve potato late blight management. Crop Protection 2020, 134. doi:10.1016/j.cropro.2020.105186.

14. Batista, D.C.; Lima, M.A.; Haddad, F.; Maffia, L.A.; Mizubuti, E.S. Validation of decision support systems for tomato early blight and potato late blight, under Brazilian conditions. Crop Protection 2006, 25, 664-670. doi:10.1016/j.cropro.2005.09.009.

15. Ziegler, S.; Arias Segura, J.; Bosio, M.; Camacho, K. Rural connectivity in Latin America and the Caribbean: A bridge for sustainable development in a time of pandemic. 2020. p. 120.

16. Peterson, P.; Arbenz, M. Scaling up agroecology to achieve the SDGs: A political matter. Farming Matters 2018, pp. 6-9.

17. Rodriguez, C.; Dimitrova Mårtensson, L.M.; Zachrison, M.; Carlsson, G. Sustainability of Diversified Organic Cropping Systems-Challenges Identified by Farmer Interviews and MultiCriteria Assessments. Frontiers in Agronomy 2021, 3. doi:10.3389/fagro.2021.698968. 
